Bundesgesundheitsbl 2018 $61: 720-728$ https://doi.org/10.1007/s00103-018-2744-9 Online publiziert: 16. Mai 2018

(c) Der/die Autor(en) 2018

CrossMark

\section{Thomas Claßen ${ }^{1}$ Maxie Bunz ${ }^{2}$}

${ }^{1}$ Fachgruppe Gesundheitsanalysen und -prognosen, Landeszentrum Gesundheit Nordrhein-Westfalen, Bochum, Deutschland

${ }^{2}$ Fachgebiet II 1.5: Umweltmedizin und gesundheitliche Bewertung, Umweltbundesamt, Berlin, Deutschland

\title{
Einfluss von Naturräumen auf die Gesundheit - Evidenzlage und Konsequenzen für Wissenschaft und Praxis
}

\section{Einleitung}

Die Lebensumwelt beeinflusst in vielfältiger Weise Gesundheit und Wohlbefinden und damit die Lebensqualität der darin lebenden und arbeitenden Bevölkerung. Insbesondere der städtische Raum wird oftmals assoziiert mit belastenden und gesundheitsschädigenden Einwirkungen über Boden, Wasser und Luft sowie aus der baulich-technischen und der sozialen Umwelt (z.B. Lärm, Luft- und Bauschadstoffe, Altlasten, Hitzeinseln, aber auch Unfälle, soziale Isolation oder Gewalterfahrungen, sogenannte "environmental bads", vgl. [1]). Den „environmental bads" stehen gesundheitsförderliche, salutogene Ressourcen aus der Umwelt (,environmental goods") gegenüber [1]. Diese können gesundheitliche Belastungen mildern, das allgemeine Wohlbefinden und die Gesundheit der Bevölkerung erhalten, aber auch steigern. $\mathrm{Zu}$ den gesundheitsförderlichen Ressourcen zählen u.a. soziale Unterstützung durch Familie und Nachbarschaft, Quartiersidentität, gesundheitsrelevante Einrichtungen (z.B. Sport- und Fitnesseinrichtungen, Facharztpraxen, Kliniken), Bewegungsfreundlichkeit von Räumen (Walkability) sowie Natur- und Landschaftselemente (vgl. [2]). Gerade in städtischen Räumen, in denen in Deutschland etwa $75 \%$ der Bevölkerung leben, sind zahlreiche dieser Ressourcen stark entwickelt.
Die unterschiedlichen Faktoren der Lebensumwelt können als sogenannte Gesundheitsdeterminanten in verschiedenartiger Weise auf unsere physische, psychische und soziale Gesundheit einwirken (vgl. u.a. [3]):

- direkt über unmittelbare Wechselwirkungen mit Individuen (z. B. Inhalation von Feinstaub, Lärmexposition oder Blick ins Grüne),

- indirekt durch die Beeinflussung weiterer Umweltfaktoren (z. B. Minderung von Hitzeextremen im urbanen Raum durch Gewässer [Stadtblau] und Grünflächen [Stadtgrün]),

- indirekt durch eine mögliche Beeinflussung des Gesundheitsverhaltens von Individuen sowie unterschiedlichen Bevölkerungsgruppen (z. B. Förderung von Bewegung).

Viele dieser Determinanten sind steuerund planbar, sind aber stets von der individuellen bis zur globalen Ebene auch in ihrer räumlichen und gesellschaftspolitischen Dimension zu betrachten ([4], siehe - Abb. 1).

In den vergangenen zwei Jahrzehnten ist die gesundheitliche Bedeutung von Naturräumen, d.h. solchen Räumen im städtischen wie auch ländlichen Raum, die vor allem durch "grüne" und "blaue“ Strukturen geprägt sind, in den Fokus von Wissenschaft und Forschung sowie der Politik, Planungs- und Umsetzungspraxis gerückt $[1-3,5]$. Die hohe gesellschaftspolitische Relevanz des Themas erschließt sich vor dem Hintergrund aktueller, weltweit wirksamer Phänomene wie

- dem Klimawandel und der Notwendigkeit adäquater Anpassungsstrategien,

- der Globalisierung und dem Druck auf Naturräume,

- dem demografischen Wandel und veränderten Bedürfnissen und Nutzungsmustern einer älteren und bunteren Gesellschaft im Hinblick auf Naturräume,

- der fortwährenden Land-StadtWanderung sowie Reurbanisierung der Innenstädte,

- der urbanen Wohnraumverknappung und des dadurch entstehenden Drucks auf Freiflächen und letztlich

- der Forderung einer nachhaltigen und gesundheitsförderlichen Entwicklung von Räumen und insbesondere Stadtregionen.

In diesem Zusammenhang wird stetig eine positive Assoziation zwischen der Aneignung (d.h. Wahrnehmung, Bewertung und Nutzung) von Naturräumen und der gesundheitlichen Lebensqualität postuliert. Oft steht pauschal die Forderung im Raum, im Rahmen einer integrierten, zukunftsfähigen Stadtentwicklungsplanung insbesondere gesundheitsförderliche Naturräume $\mathrm{zu}$ erhalten, zu erweitern und ggf. wiederherzustellen (vgl. u. a. $[6,7]$ ). Doch welche konkreten Wirkzusammenhänge 


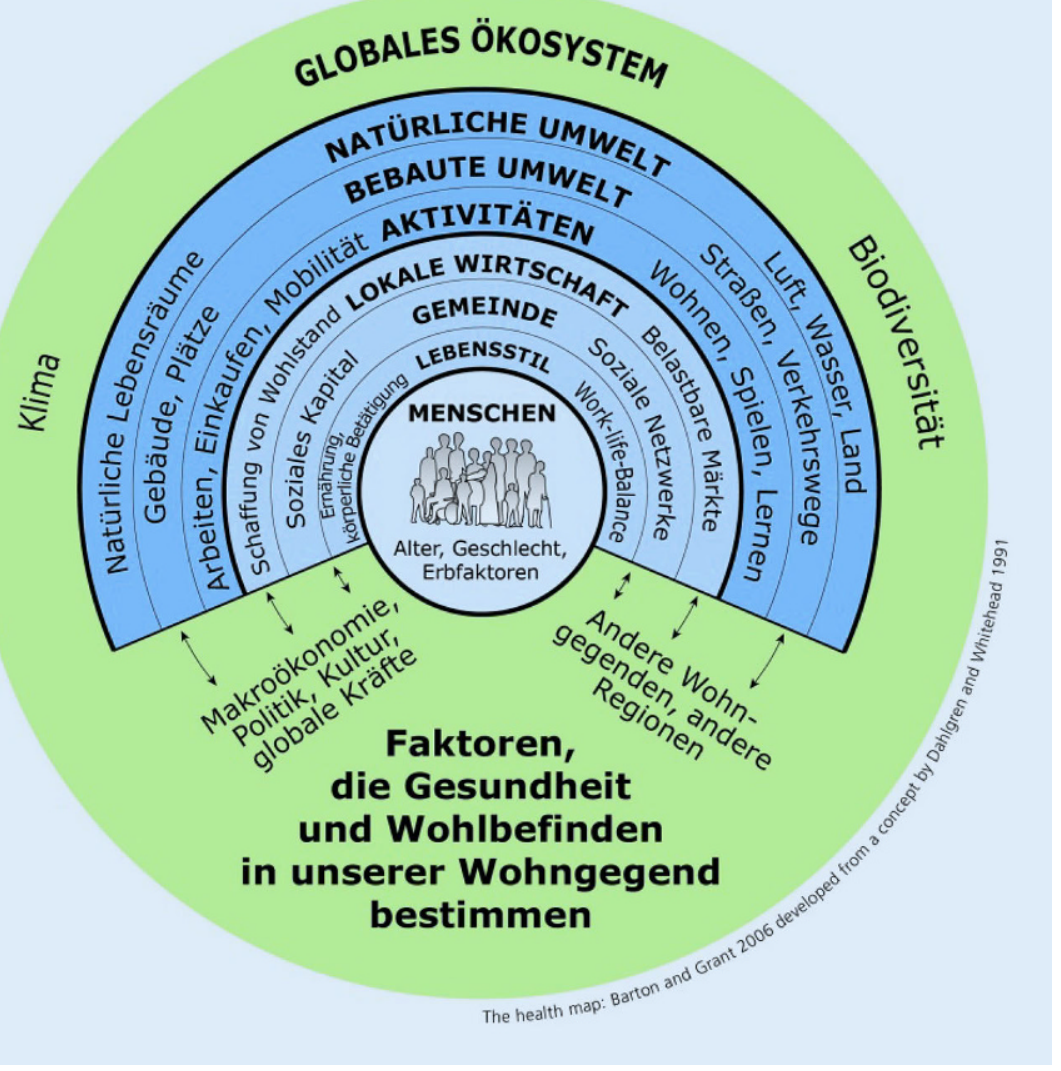

Abb. 1 ॥ Humanökologisches Modell der Gesundheitsdeterminanten im Siedlungsraum, auch Health Map genannt (autorisierte Übersetzung nach Barton \& Grant [4]). Abbildung mit freundl. Genehmigung $\odot$ SAGE Publications on behalf of Royal Society for Public Health

bestehen zwischen Gesundheit, Wohlbefinden und Naturräumen, und welche Konsequenzen ergeben sich daraus für Wissenschaft, Politik und Planung? Der folgende Übersichtsbeitrag liefert hierzu einige Antworten.

\section{Naturräume und Gesundheit}

Die gesundheitsschützende ebenso wie die gesundheitsfördernde Wirkung von Naturräumen allgemein sowie von Stadtgrün und Stadtblau im Besonderen ist vielfach belegt (u. a. $[8,9])$. Nachfolgend wird die diesbezügliche aktuelle Evidenzlage dargestellt. Mögliche gesundheitsgefährdende Wirkungen von Naturräumen werden ebenfalls kurz thematisiert, sind jedoch nicht Schwerpunkt dieses Beitrags. $\square$ Abb. 2 liefert einen Überblick der im weiteren Beitrag ausgeführten Aspekte. den größeren Abstand zwischen Lärmquelle und lärmexponierten Personen sowie durch eine geringere Schallreflektion und stärkere Streuung aufgrund der vergleichsweise rauen Oberfläche. Die faktische Pegelminderung kann in Ausnahmefällen 3-5dB betragen, z. B. bei begrünten Bahngleisen (Rasengleisen) mit hochliegender Vegetationsdecke, wird insgesamt jedoch häufig überschätzt (u.a. [13]). Für die subjektiv wahrgenommene Lautstärkenminderung ist auch entscheidend, dass Grünräume und Gewässer durch die selbst erzeugte Geräuschkulisse eine positiv bewertete Soundscape (z.B. Blätterrauschen, Vogelgezwitscher, Wasserplätschern) erzeugen. Damit überlagern sie zum Teil den störenden Umgebungslärm, mindern die subjektiv empfundene Lärmbelästigung der Bevölkerung und steigern so die Aufenthaltsqualität [14]. Dieser Effekt wird durch die visuelle Abschirmung der Lärmquelle sogar noch verstärkt [14].

Aus klimaökologischer Sicht sind Grün- und Gewässerstrukturen in mehrfacher Weise gesundheitsschützend wirksam. So können sie durch die Transpirationsleistung (u.a. Erhöhung der absoluten Luftfeuchtigkeit), die raue Oberfläche natürlicher, bewachsener und feuchter Böden (mit geringerer Wärmespeicherung als asphaltierte Flächen) und die Schattenwirkung des Kronendachs von Bäumen erheblich dazu beitragen, die Temperatur an heißen Tagen, insbesondere in hitzebelasteten urbanen Räumen (Hitzeinseln), zu senken [15]. Wasserflächen besitzen ein eigenständiges Ausgleichspotenzial mit Kühlungseffekten, die mit ca. 2,5 K sogar noch deutlich über der Reduktionsleistung von Grünräumen liegen können [16]. In der kälteren Jahreszeit hingegen wirken offene Wasserflächen als Wärmespeicher [16]. Mit der Zunahme von Hitzetagen und Tropennächten infolge des erwarteten Klimawandels gewinnen Naturräume in der Stadt als Klimakomfortinseln insbesondere für besonders vulnerable Bevölkerungsgruppen erheblich an Bedeutung [17]. Weiterhin können Grünflächen, z. B. in Form von Parks, größere Wassermassen aufnehmen und den Oberflächenabfluss im städtischen Gebiet reduzieren (vgl. [18]). 
Somit können sie auch als Überschwemmungsschutz bei Starkregenereignissen dienen.

\section{Gesundheitsfördernde Potenziale von Naturräumen}

Einige Studien und Übersichtsarbeiten gaben schon vor der Jahrtausendwende Hinweise auf eine Steigerung des allgemeinen (gesundheitlichen) Wohlbefindens der Bevölkerung durch die Nutzung von Naturräumen (vgl. u.a. [19-22]), auch wenn die Quantität und Qualität des betrachteten „Grüns" häufig nicht systematisch erfasst wurde. Inzwischen gibt es aber eine Vielzahl an Studien, die direkte und indirekte positive Einflüsse von Naturräumen auf das Wohlbefinden von Individuen und Bevölkerungsgruppen betrachten $[3,8,9]$. Hierbei spielt auch der Typus des Naturraums (naturnah oder stark anthropogen überformt, Wald oder Park, vgl. [23]) eine Rolle. An dieser Stelle ist jedoch der Hinweis angezeigt, dass die Erkenntnisse zumeist aus epidemiologischen Querschnittstudien mit teils sehr unterschiedlichen Naturraumdefinitionen stammen und somit Assoziationen, aber keine gesicherten Wirkungen beschreiben. In experimentellen Studien werden hingegen häufig typisch urbane Szenerien eher ländlichen, naturnahen Szenerien gegenübergestellt. Nachfolgend wird die Evidenzlage zu Zusammenhängen zwischen Naturräumen und mentalem, physischem und sozialem Wohlbefinden dargestellt, wobei eine strikte Trennung schwierig ist, da die Übergänge gerade zwischen Psyche und Physis fließend sind.

\section{Mentales Wohlbefinden}

Im Hinblick auf das mentale (psychische) Wohlbefinden, das stark von der individuellen Raumwahrnehmung, -bewertung und -konstruktion beeinflusst wird, sind Naturräume über die Komponente des Erlebens besonders wirksam (u.a. [22]). So konnte gezeigt werden, dass das Natur- und Landschaftserlebnis eine stressreduzierende, blutdrucksenkende, aufmerksamkeitserhöhende, konzentrationssteigernde und restorati-

Bundesgesundheitsbl 2018 $\cdot 61: 720-728$ https://doi.org/10.1007/s00103-018-2744-9

(c) Der/die Autor(en) 2018

\section{T. Claßen · M. Bunz}

\section{Einfluss von Naturräumen auf die Gesundheit - Evidenzlage und Konsequenzen für Wissenschaft und Praxis}

\section{Zusammenfassung}

Naturräumen und insbesondere urbanen Grünräumen (Stadtgrün) und Gewässern (Stadtblau) wird seit Langem ein großes gesundheitsschützendes und -förderndes Potenzial beigemessen. Sie können in vielfältiger Weise positiv auf die psychische, physische und soziale Gesundheit sowie das Wohlbefinden von Menschen einwirken direkt oder indirekt: direkt durch die Minderung und Moderation möglicher Risiken aus der Lebensumwelt (z. B. Lärm, Feinstaub, Hitze), durch die psychisch-physiologischen Wirkungen des Naturerlebnisses und die physischen Auswirkungen der Exposition gegenüber Naturstoffen und -elementen; indirekt wirken Naturräume beispielsweise über die Anregung gesundheitsförderlicher Verhaltensweisen (z. B. Anreiz für mehr Bewegung), über die Nutzung als Outdoortreffpunkt sowie die Minderung von Aggressionen und daraus resultierender Effekte für das soziale Wohlbefinden. Einzelne mögliche gesundheitsabträgliche
Wirkungen von Naturräumen sind ebenfalls beschrieben worden (z. B. Unsicherheit oder Angst in unübersichtlicher Natur, mögliche Nutzungskonkurrenzen, Allergien und Hautirritationen durch Naturelemente, Infektionsrisiken durch Wirtstiere). Vor dem Hintergrund der positiven Wirkungen von Naturräumen wird bisweilen gefordert, diese im Rahmen einer integrierten, zukunftsfähigen Entwicklung von Kommunen zu erhalten, zu fördern und gegebenenfalls wiederherzustellen. Doch welche konkreten Wirkzusammenhänge bestehen zwischen Naturräumen und Gesundheit? Welche Forderungen und Perspektiven ergeben sich für eine gesundheitsförderliche Umsetzungspraxis? Mit diesen Fragen setzt sich der Übersichtsbeitrag auseinander und liefert einige Antworten.

Schlüsselwörter

Natur - Gesundheit · Stadtgrün · Stadtblau . Wohlbefinden

\section{Contribution of natural spaces to human health and wellbeing}

\section{Abstract}

Natural spaces and especially urban green and blue spaces have been recognised for a long time as spaces with great potential for protecting and promoting human health and well-being. They may affect human physical, mental and social health and well-being in various ways. On one hand, this comes to pass through reduction and moderation of potential environmental health risks (e.g. noise, particulate matter, heat), psychophysiological effects of nature experience, as well as physical effects of exposure to natural compounds and elements. On the other hand, natural spaces can affect health and well-being indirectly e. g. through motivation of health promoting behaviour (e.g. more physical activity) and through use as outdoor meeting spaces, by decreasing aggression, and through the resulting positive effects on social well-being. Yet, some potential adverse health effects of nature and landscapes have been reported, too (e. g. insecurity or fear in confusing or unmaintained natural spaces, potential rivalry in usage, allergies or skin irritations due to natural elements, risk of communicable diseases from vectors). Against the background of positive effects of natural spaces, creating, restoring and enhancing urban green and blue spaces are often claimed in terms of sustainable and integrated urban development. But which associations and impacts exist between natural spaces and health? What are the resulting demands when integrating natural spaces for a health-promoting implementation practice? This overview article provides some answers to these questions.

Keywords

Nature · Health · Urban green spaces · Urban blue spaces · Well-being 


\section{Gesundheitsförderndes Potenzial}

Mental
- Reduktion von Stressempfinden
- Erholung der
Aufmerksamkeitskapazität
- Steigerung von positivem Affekt
- Verringerung psychischer Belastung
- Positive Effekte bei bestehenden
psychischen Störungen

- Lärmminderung und -moderation

- Schadstofffilterung und -umwandlung

- Klimaökologischer Ausgleich

- Abmilderung von Hitze- und Kälteextremen

- Überschwemmungsschutz

\section{Körperlich}

- Verringertes Morbiditäts- und Mortalitätsrisiko für bestimmte Erkrankungen

- Anreiz zusätzlicher physischer Outdooraktivität

- Senkung von Blutdruck und

Stresshormonexpression

- Assoziation mit neuronaler Integrität

\section{Sozial}

- Möglichkeit zu Begegnung und sozialem Austausch

- Chancen für Integration und Inklusion

- Verringerung gesundheitlicher

Benachteiligung für Menschen mit niedrigem sozioökonomischen Status

\section{Gesundheitsschützendes Potenzial}

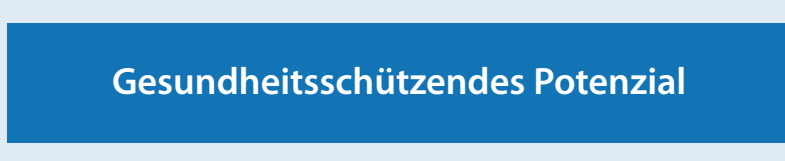

\section{.}

Abb. 2 \ Überblick über die potenziellen gesundheitlichen Wirkungen von Naturräumen

ve Wirkung haben kann (siehe folgende Abschnitte, vgl. u. a. [9]). Insbesondere die Ergebnisse zur Aufmerksamkeitsund Konzentrationssteigerung gehen konform mit der Attention-RestorationTheory [19], nach der es in der Natur $\mathrm{zu}$ einer kognitiven Erholung kommt. Zudem gibt es mittlerweile Hinweise aus der Forschung, die einen positiven Zusammenhang zwischen der langfristigen Exposition gegenüber Grünräumen und der kognitiven Entwicklung von Kindern sowie der kognitiven Funktion Erwachsener nahelegen [24].

Neben der Bedeutung für Aufmerksamkeit und kognitive Funktionen haben Naturräume u. a. einen Einfluss auf Emotionen, psychische Belastungen und Stressempfinden. Hartig et al. [25] konnten nachweisen, dass durch einen Spaziergang in der Natur der positive Affekt zunahm, während Ärger abnahm, verglichen mit einem Spaziergang entlang einer urbanen Straße. Auch Roe und Aspinall [26] konnten in einer quasiexperimentellen Studie mit zwei Gruppen (eine mit guter mentaler Verfassung, eine mit einer klinisch diagnostizierten psychischen Störung) zeigen, dass der Spaziergang in ländlicher Gegend sich für beide Gruppen positiv auf die Stimmung und das Stresserleben auswirkte. Darüber hinaus führte der Spaziergang im ländlichen Raum zu einem höheren restorativen Effekt in der Gruppe derer mit psychischer Störung [26]. Dies weist auf das Potenzial von Naturräumen als Coping-Ressource bei bestehenden psychischen Störungen hin, zum einen aufgrund der restorativen Wirkung, zum anderen durch die Förderung körperlicher Bewegung und sozialer Kontakte bei gemeinschaftlichen Aktivitäten (s. unten).

In weiteren Studien konnte gezeigt werden, dass ein höheres Maß an Grün im Wohnumfeld mit geringerem Auftreten von Angststörungen, geringerem Stresserleben und weniger depressiven Symptomen assoziiert ist $[9,27,28]$. Längsschnittevidenz kommt von Alcock et al. [29], in deren Studie sich die selbstbewertete psychische Gesundheit von Studienteilnehmenden nach einem Umzug in eine grünere Gegend langfristig verbesserte.

Vereinzelte Studien geben auch Hinweise darauf, dass eine wahrgenommene hohe Biodiversität im Vergleich mit Flächen geringerer Biodiversität positiv assoziiert ist mit einem gesteigerten mentalen Wohlbefinden (vgl. u. a. [30]).

Mit dem Erlebnis von Gewässerstrukturen wird in vereinzelten Studien unabhängig von Gewässertypus und -größe zudem eine über die Wirkung reiner Grünräume hinausgehende erholsame, stressmildernde Wirkung assoziiert $[31,32]$. Jedoch gilt es auch, die Art von Grünräumen zu berücksichtigen: Insbesondere Waldgebieten wird ein positiver Einfluss auf die Stresslinderung und die Stimmung zugeschrieben sowie eine verminderte Aktivität des sympathischen Nervensystems (vgl. [33, 34]). Kühn et al. [35] stellten in einer Untersuchung älterer Menschen in Berlin eine signifikant bessere strukturelle Integrität der Amygdala bei Probanden fest, die nah am (Stadt-)Wald wohnen, im Vergleich zu solchen, die nah am Wasser oder sonstigen Grünräumen wohnen. Dies könnte auf eine höhere Stressresilienz bei Menschen hinweisen, die in der Nähe eines Waldgebiets wohnen. Da die Stressreaktion und die Funktionsweise der Amygdala eine Rolle in der Entstehung bestimmter psychischer Störungen wie der Depression spielen, könnten die Ergebnisse ein Indiz für ein vermindertes Ri- 
siko des Auftretens von durch neuronale Prozesse ausgelösten psychischen Störungen darstellen. An den aufgeführten Studienergebnissen zeigt sich auch die enge Verquickung von psychischer und physischer Gesundheit über physiologische Prozesse.

\section{Körperliches Wohlbefinden}

In Studien zu den direkten Wirkungen von Natur und Stadtgrün auf die physische Gesundheit wurden mögliche Effekte auf die Mortalität, die Morbidität sowie kurzzeitige körperliche Reaktionen, wie beispielsweise eine verminderte Expression von Stresshormonen, beschrieben.

Erste statistisch abgesicherte Hinweise $\mathrm{zu}$ direkten Wirkungen von Naturräumen auf die physische Gesundheit finden sich in den 1980er-Jahren. In einer kleinen, aber vielbeachteten Studie untersuchte Ulrich [36] retrospektiv in einem quasiexperimentellen Design den Heilungsprozess von stationären Patienten nach Gallenblasenentfernung. Bei ansonsten vergleichbaren Bedingungen machte der Blick aus dem Fenster den Unterschied. Eine Gruppe schaute auf eine Baumgruppe, die andere Gruppe hingegen auf eine gegenüberliegende Backsteinwand. Die Ergebnisse zeigten, dass diejenigen Personen mit Blick auf die Baumgruppe u.a. früher entlassen wurden und deutlich weniger Schmerzmittel einnahmen [36].

Hartig et al. [25] zeigten die Wirkung des Naturerlebnisses auf den Blutdruck, welchen sie als Indikator für das Stresslevel der Probanden nutzen. Der diastolische Blutdruck sank in einer Probandengruppe während des Spaziergangs durch einen ländlichen Naturraum $a b$, während dieser in der Gruppe mit Spaziergang entlang der Straße nach kurzzeitigem Absinken wieder deutlich anstieg [25]. Zwischenzeitig sind zahlreiche Studien durchgeführt worden, die die Ergebnisse von Hartig et al. [25] stützen. Stets geht die Exposition gegenüber natürlichen Umgebungen einher mit einer Absenkung des Blutdrucks sowie des Cortisolspiegels und weiterer Stresshormone als Indikator für ein reduziertes Stressniveau (vgl. u. a. [9]).
Auch Li et al. [37] bestätigten in verschiedenen Studien, in der die Wirkung des längeren Aufenthalts im Wald (das sogenannte Forest Bathing oder ShinrinYoku) analysiert wurde, den Abbau der Stresshormone Adrenalin und Noradrenalin. Darüber hinaus wiesen sie auch eine krebspräventive Wirkung durch den Aufbau sogenannter Krebskillerzellen („human natural killer activity“) sowie die Ausschüttung von intrazellulären Krebsabwehrproteinen nach ([37], vgl. auch [38]). Als Erklärung wird die erhöhte Konzentration von Phytonziden (pflanzliche Biozide) in der Waldluft diskutiert.

Maas et al. [28] konnten in einer niederländischen Untersuchung zeigen, dass Menschen, die im städtischen Umfeld mit höherem Grünanteil in der direkten Nachbarschaft (1 km Radius) leben, signifikant seltener an kardiovaskulären, muskuloskelettalen, psychischen, respiratorischen, neurologischen und intestinalen Krankheitsbildern sowie weiteren Erkrankungen wie Diabetes leiden als Personen, die einen geringeren Grünanteil im Wohnumfeld haben oder in größerer Entfernung zu Grünräumen leben. In dieser Studie wurden Grünräume jedoch sehr weit gefasst und enthielten auch landwirtschaftliche Nutzflächen.

Ergebnisse einer Studie aus Japan von Takano et al. [39] weisen darauf hin, dass eine gute Versorgung mit und ein guter Zugang zu städtischen Grünflächen signifikant positiv mit Lebensqualität und Lebenserwartung älterer Menschen assoziiert ist.

Mitchell und Popham [40] fanden in einem englischen Forschungsprojekt zudem weniger Ungleichheiten hinsichtlich Herz-Kreislauf-Erkrankungen und Mortalität zwischen Menschen mit hohem und niedrigem sozioökonomischen Status in grüneren Gegenden als in Gebieten mit einem geringen Zugang zu natürlichen Grünräumen. Dies ist auch deshalb bedeutsam, da die Verteilung von qualitativ hochwertigen und sicheren Grünflächen meist zuungunsten von Gruppen mit niedrigem sozioökonomischen Status sowie ethnischen Minderheiten ausfällt [41].

Untersuchungen von Richardson und Mitchell [42] zu möglichen geschlechts- spezifischen Unterschieden ergaben, dass die Mortalitätsraten durch kardiovaskuläre und respiratorische Erkrankungen bei Männern umso geringer waren, je größer der Grünanteil war. Diese Assoziation ließ sich jedoch für Frauen nicht nachweisen. Dies erklärten die Autoren mit der geschlechterdifferenzierten Wahrnehmung und Nutzung von Grünräumen.

In den vergangenen Jahren konnten zudem verschiedene Querschnittstudien in Spanien und Deutschland einen Zusammenhang zwischen dem Grünraumanteil in der Wohnumgebung und dem Geburtsgewicht von Neugeborenen identifizieren ([43]; vgl. [9, 44]). Nach Adjustierung für Luftverunreinigungen, Abstand zu stark befahrenen Straßen, Bevölkerungsdichte und Bildungsgrad verstärkte sich der statistische Zusammenhang [43]. Erklärungsversuche sehen eine höhere Grundzufriedenheit und Aktivität der Schwangeren als moderierende Faktoren.

\section{Anreiz für körperliche Aktivität}

Immer wieder steht die Frage im Raum, inwieweit Naturräume insbesondere in urbanen Gebieten Anreiz und Motivation für eine verstärkte körperliche Aktivität (alltägliche Bewegung, Sport) bieten und somit indirekte Gesundheitseffekte erwarten lassen im Hinblick auf die Stärkung des Herz-Kreislauf- und Immunsystems sowie zur Prävention zahlreicher Zivilisationskrankheiten (z. B. Adipositas, Bluthochdruck, Diabetes mellitus Typ II oder Rückenschmerzen). Frank et al. [45] konnten für Bielefeld zeigen, dass für $71 \%$ der Befragten Bewegung der Grund für das Aufsuchen von Grünräumen ist. Ähnliches konnten Völker und Kistemann [32] auch für den Aufenthalt an Gewässern nachweisen. Fraglich ist jedoch, ob ein Mehr an Naturräumen auch ein Mehr an Bewegung bedingt. Maas et al. [46] konstatieren, dass der Grad der physischen Aktivität kaum mit einer grünen Umgebung in Beziehung steht und der Anteil der physischen Bewegung in einer grüneren Umgebung den Zusammenhang zwischen Grünräumen und selbstwahrgenommener Gesundheit nicht erklären kann. Ebenso wiesen Dad- 
vand et al. [47] darauf hin, dass der $\mathrm{Zu}$ sammenhang zwischen subjektiver Gesundheit und Grünflächen in Barcelona zwar u.a. durch den psychischen Gesundheitsstatus und die wahrgenommene soziale Unterstützung mediiert werden, allerdings nur in sehr geringem Maße durch die physische Aktivität, wobei hier jedoch nicht zwischen Indoor- und Outdooraktivität unterschieden wurde. De Vries et al. [48] konnten hingegen in einem Review anhand zahlreicher Studien Anreiz und Motivation von einer grünen Umgebung zu zusätzlicher Bewegung feststellen, beschreiben jedoch auch, dass die Evidenz gemischt ist und es an ausreichenden, qualitativ hochwertigen Studien mangelt.

\section{Soziales Wohlbefinden}

Als öffentliche und frei zugängliche Begegnungsräume wirken sich Naturräume vor allem in der Stadt auch positiv auf das soziale Wohlbefinden der Menschen aus [49, 50]. Denn hinsichtlich der Möglichkeiten, soziale Kontakte zu schließen und zu pflegen, gelten urbane Grünräume und Gewässerufer als bedeutsame Begegnungsstätten [50, 51], die zudem als Identifikationsorte eine große Symbolkraft entfalten können [32]. Man denke hier z. B. an den Central Park in New York, den Berliner Tiergarten oder die Kölner Rheinpromenade. Insbesondere in Parks erfolgen eine Durchmischung sowie ein Nebeneinander unterschiedlichster sozialer Gruppen (z. B. in Bezug auf Alter, Geschlecht, ethnische Herkunft). Damit besitzen städtische Naturräume ein nicht zu unterschätzendes Potenzial zur sozialen Integration, Inklusion und Akzeptanzsteigerung [50] und können im Wohnumfeld als unbelasteter Begegnungs- und Kommunikationsraum den sozialen Zusammenhalt der dort ansässigen Bevölkerung stärken [52]. Dies spiegelt sich auch in aktuellen Bewegungen wie Urban Gardening wider (u. a. [53]). Darüber hinaus verfügen Grünräume auch über das Potenzial, der Kriminalitätsentstehung beispielsweise in sozial benachteiligten Stadtgebieten entgegenzuwirken, sofern sie offen und einladend gestaltet sind und damit zum häufigeren Aufenthalt draußen im
Sinne eines „Outdoorwohnzimmers“ anregen $[9,51,52]$. Im Falle konkurrierender Nutzungsinteressen (Grillen, Skaten, Radfahren, Joggen, Spielen, Lesen, Ruhen etc.) unterschiedlicher Gruppen in kleinflächigen Grünräumen und auf grünen Wegeverbindungen können indes auch Konflikte entstehen (vgl. [54]).

\section{Einfluss der Qualität von Naturräumen}

Wie aus den vorangehenden Abschnitten ersichtlich wurde, können Naturräume insgesamt, aber insbesondere urbane Grünräume und Gewässer verschiedenste Funktionen ausüben im Hinblick auf gesundheitsschützende und gesundheitsfördernde Wirkungen (siehe • Abb. 2).

Die bisher genannten Studien haben gemeinsam, dass sie vornehmlich die Quantität von Naturräumen im Studiendesign zugrunde legten, nicht jedoch qualitative Aspekte. Van Dillen et al. [55] und de Vries et al. [56] konnten hingegen in einer Untersuchung in 80 niederländischen Wohnquartieren zeigen, dass sowohl die Verfügbarkeit als auch die Qualität von Grünräumen und Straßenbegleitgrün positiv mit der selbstbewerteten Gesundheit der Studienteilnehmenden assoziiert waren, wobei diese für Straßenbegleitgrün besonders ausgeprägt waren. Auch in einer australischen Studie wurde deutlich, dass im urbanen Raum nicht allein die Quantität von Grünräumen ausschlaggebend, sondern eine hohe Qualität mit einem niedrigeren psychosozialen Stresslevel assoziiert ist [57].

\section{Gesundheitsgefährdende Wirkungen}

Über das zuvor beschriebene soziale Konfliktpotenzial hinaus gibt es auch Evidenz zu verschiedenen möglichen gesundheitsabträglichen Wirkungen von Naturräumen. So können, entgegen der sonst anzunehmenden Verbesserung der Luftqualität durch Grünelemente, beispielsweise geschlossene Kronendächer in städtischen Alleen lufthygienische Problemlagen verstärken [11]. Weiterhin konnten Churkina et al. [58] zeigen, dass einige flüchtige organische Ver- bindungen, die von der Vegetation bei hohen Temperaturen verstärkt emittiert werden, in Kombination mit hohen Konzentrationen an Stickstoffoxiden an sehr heißen Tagen bis zu $60 \%$ zur Bildung von bodennahem Ozon beitragen können.

Darüber hinaus konnten einzelne Studien nachweisen, dass die These der Biophilie als einer Menschen innewohnenden Liebe zu allem Lebendigen nicht allgemeingültig ist, sondern z.B. im Ekel oder gar der Angst vor Spinnen, Mäusen usw. ihre deutlichen Grenzen findet (vgl. [59]). Weiterhin können negative Gefühle durch unübersichtliche, schlecht einsehbare Naturräume, wie dichte Wälder oder verwinkelte Parks mit hohen Gebüschen und mangelnder Beleuchtung, gerade in der Dämmerung hervorgerufen werden. Hiermit assoziierte Ängste sind ebenso beschrieben wie ein erhöhtes Risiko für Kriminalitätsförderung (z. B. Drogenkriminalität, Überfälle, Gewalt gegen Frauen, vgl. [9, 51]).

Ein weiteres Risiko geht mit Allergien oder Hautirritationen durch Naturelemente wie Pollen (z. B. durch Birken, Erlen), Tierhaare (z. B. Eichenprozessionsspinnerraupen [60]) oder Pflanzensäften (z.B. Herkulesstauden) einher (vgl. [9]), die große Bevölkerungsanteile betreffen können. So gaben in der deutschlandweiten GEDA-Studie 28,1\% der Erwachsenen an, aktuell von Allergien betroffen zu sein [61]. Auch wenn nicht eindeutig ist, in welchem Maße Grünflächen zur Allergieauslösung beitragen [62], sollte bei einer Neupflanzung von Bäumen im öffentlichen Raum auf die Anpflanzung solcher Baumarten, die im Zusammenhang mit einer weiteren Erhöhung der Baumpollenzahl mit allergenem Potenzial stehen, verzichtet werden [63].

Schließlich sind auch Risiken durch Infektionskrankheiten $\mathrm{zu}$ nennen, die mit Wirtstieren assoziiert sind, welche an Grün- und Gewässerstrukturen gebunden sind (z.B. Zecken, Mücken, Nagetiere).

Bei der Gestaltung von Naturräumen, die auch eine wesentliche Erholungsfunktion erfüllen sollen, sind diese Aspekte in besonderer Weise zu bedenken. 


\section{Umsetzung in der Praxis: Anforderungen an "gute" Naturräume}

Naturräume gelten heutzutage aufgrund der vielfältigen gesundheitsschützenden und gesundheitsförderlichen Wirkungen als wichtige Gesundheitsdeterminante und als wesentlicher Baustein der Daseinsvorsorge. Auf Grundlage der erwähnten Studienergebnisse wird zunehmend in Wissenschaft und Praxis diskutiert, wie Naturräume (vor allem in Städten) beschaffen sein sollten, um ihr gesundheitsförderliches Potenzial bestmöglich entfalten zu können. Hierbei haben sich folgende Kriterien und Merkmale als besonders wichtig herauskristallisiert (vgl. [54]):

- gute Verfügbarkeit, Erreichbarkeit und Zugänglichkeit von Naturräumen, möglichst ohne Notwendigkeit der Nutzung eines Autos und insbesondere für Bevölkerungsgruppen, die keinen Zugang zu einem privaten Garten haben,

- gleichmäßige und sozial gerechte Verteilung und Vernetzung von Naturräumen,

- Möglichkeiten zur Aufnahme und Pflege von Kontakten (z.B. für Eltern mit kleinen Kindern und ältere Menschen), aber auch zur Beobachtung von Tieren und anderen Menschen,

- multifunktionale Nutzung mit ausgewogenem Verhältnis von Räumen für Erholung, Erlebnis, Bewegung, individuelle Ruhe und soziale Interaktion,

- Vermeidung von Angsträumen und Mobilitätsbarrieren (z. B. Reduzierung von Verletzungsrisiken, Beleuchtung von Hauptwegen, Übersichtlichkeit),

- Kombination unterschiedlicher Aspekte von Naturräumen im urbanen Raum insbesondere dort, wo größere Naturräume Mangelware sind (z. B. Fassaden- und Dachbegrünungen, Wasserspiele),

- ästhetisch ansprechende Landschaften.

Über diese Kriterien und Merkmale hinaus sollten die verschiedenen Wahr- nehmungs- und Aneignungsmuster unterschiedlicher soziodemografischer und kultureller Gruppen in Bezug auf Naturräume ebenso wie sozialräumliche Problemlagen in der Bevölkerung bei der Naturraumentwicklung stets berücksichtigt werden. Denn gerade sozial benachteiligte Gebiete sind aufgrund ihrer Lage und verminderten Wohnraumqualität oftmals mehrfach belastet z. B. im Hinblick auf Luftverunreinigungen, Lärm oder sommerliche Hitze und weisen zudem eine vergleichsweise geringere Verfügbarkeit und Qualität von Naturräumen auf [54].

In bisherigen Studien zur gesundheitlichen Bedeutung von Naturräumen wurde ein deutlicher Fokus auf die Bedingungen in urbanen Räumen gelegt. Studien, die explizit gesundheitliche Wirkungen von Naturräumen in urbanen und ländlichen Gebieten einander gegenüberstellen, sind nicht bekannt. Hinsichtlich des Unterschieds der Wirkungen von ländlichen und städtischen Naturräumen auf die menschliche Gesundheit besteht daher ein erheblicher Forschungsbedarf.

Naturräume in der Stadt sind zwar kein Allheilmittel, und auch sogenannte graue Strukturen (gebaute Strukturen wie Häuserzeilen und Plätze), die architektonisch ansprechend sind, können ausgezeichnete Destinationen für Gesundheit und Wohlbefinden sein. Aber in der Stadt wie auch in Stadtrandlagen und im ländlichen Raum gilt es, den Natur- und Landschaftsraum als begrenzte und gefährdete Ressource auch für eine hohe gesundheitliche Lebensqualität der Bevölkerung zu begreifen und entsprechend zu fördern. Gefordert sind für die Umsetzung dieses Ziels unterschiedlichste Akteure aus Wissenschaft, Politik und Praxis [5, 10, 64, 65]. Umso wichtiger ist eine ressortübergreifende Vernetzung der Sektoren Kommunalplanung, Gesundheit, Umwelt und Soziales im Sinne integrierten Politik- und Verwaltungshandelns sowie der Mut zu inter- und transdisziplinären Forschungsund Praxiskooperationen auf dem Weg in eine gesundheitsförderliche Zukunft für alle.

\section{Korrespondenzadresse}

Dr. T. Claßen

Fachgruppe Gesundheitsanalysen und -prognosen, Landeszentrum Gesundheit Nordrhein-Westfalen

Gesundheitscampus 10, 44801 Bochum, Deutschland

thomas.classen@lzg.nrw.de

\section{Einhaltung ethischer Richtlinien}

Interessenkonflikt. T. Claßen und M. Bunz geben an, dass kein Interessenkonflikt besteht

Dieser Beitrag beinhaltet keine von den Autoren durchgeführten Studien an Menschen oder Tieren.

Open Access. Dieser Artikel wird unter der Creative Commons Namensnennung 4.0 International Lizenz (http://creativecommons.org/licenses/by/4.0/deed. de) veröffentlicht, welche die Nutzung, Vervielfältigung, Bearbeitung, Verbreitung und Wiedergabe in jeglichem Medium und Format erlaubt, sofern Sie den/die ursprünglichen Autor(en) und die Quelle ordnungsgemäßnennen, einen Linkzur Creative Commons Lizenz beifügen und angeben, ob Änderungen vorgenommen wurden.

\section{Literatur}

1. Frumkin $H$ (2003) Healthy places: exploring the evidence. Am J Public Health 93:1451-1456

2. Claßen T, Völker S, Baumeister H et al (2014) Welchen Beitrag leisten urbane Grünräume (Stadtgrün) und Gewässer (Stadtblau) für eine gesundheitsförderliche Stadtentwicklung? Einblicke in die Arbeit der Juniorforschungsgruppe "StadtLandschaft \& Gesundheit". UMID Umwelt Mensch Informationsd 02/2014:30-37

3. Hartig T, Mitchell R, De Vries S, Frumkin H (2014) Nature and health. Annu Rev Public Health 35:207-228

4. Barton $\mathrm{H}$, Grant $M$ (2006) A health map for the local human habitat.JRSocPromot Health 126:252-253

5. Rittel K, Bredow L, Wanka ER et al (2014) Grün, natürlich, gesund: Die Potenziale multifunktionaler städtischer Räume. BfN, Bonn

6. Dannenberg AL, Frumkin H, Jackson RJ (Hrsg) (2011) Making healthy places: designing and building for health, well-being, and sustainability. Island Press, Washington D.C.

7. Rodenstein M (2012) Stadtplanung und Gesundheit: Ein Rückblick auf Theorie und Praxis. In: Böhme C, Kliemke C, Reimann B, Süß W (Hrsg) Stadtplanung und Gesundheit. Huber, Bern, S15-26

8. Hornberg C, Beyer R, Claßen T et al (2016) Stadtnatur fördert die Gesundheit. In: Kowarik I, Bartz R, Brenck M (Hrsg) Ökosystemleistungen in der Stadt - Gesundheit schützen und Lebensqualität erhöhen. Naturkapital Deutschland - TEEB DE, Berlin, Leipzig, S98-124

9. World Health Organization Regional Office for Europe (WHO Europe) (2016) Urban green spaces and health - a review of evidence. WHO Europe, Copenhagen 
10. Kowarik I, Bartz R, Brenck M (Hrsg) (2016) Ökosystemleistungen in der Stadt - Gesundheit schützen und Lebensqualität erhöhen. Naturkapital Deutschland-TEEB DE, Berlin, Leipzig

11. Säumell,Draheim T, EndlicherW, LangnerM(2016) Stadtnatur fördert saubere Luft. In: Kowarik I, Bartz $\mathrm{R}$, Brenck M (Hrsg) Ökosystemleistungen in der Stadt - Gesundheit schützen und Lebensqualität erhöhen. Naturkapital Deutschland - TEEB DE, Berlin, Leipzig, S71-79

12. Claßen T, Jäcker-Cüppers $M$, Riedel N (2016) Stadtnatur mindert Lärm. In: Kowarik I, Bartz $\mathrm{R}$, Brenck M (Hrsg) Ökosystemleistungen in der Stadt - Gesundheit schützen und Lebensqualität erhöhen. Naturkapital Deutschland - TEEB DE, Berlin, Leipzig, S80-85

13. Yang F, Bao ZY, Zhu ZJ (2011) An assessment of psychological noise reduction by landscape plants. Int JEnviron Res Public Health 8:1032-1048

14. Coensel BD, Vanwetswinkel S, Botteldooren D (2011) Effects of natural sounds on the perception of road traffic noise. J Acoust Soc Am 129:EL148-EL153

15. Bowler DE, Buyung-Ali L, Knight TM, Pullin AS (2010) Urban greening to cool towns and cities: a systematic review of the empirical evidence. Landsc Urban Plan 97:147-155

16. Völker S, Baumeister $\mathrm{H}$, Claßen T, Hornberg C, Kistemann T (2013) Evidence for the temperaturemitigating capacity of urban blue space - a health geographic perspective. Erdkunde 67:355-371

17. Schmidt C, Seidel A, Kolodziej J (2010) Vulnerabilitätsanalyse Westsachsen: Zwischenstand. Regionaler Planungsverband Leipzig-Westsachsen, TU Dresden, Lehr- und Forschungsgebiet Landschaftsplanung, Leipzig, Dresden

18. Breuste J, Haase D, Pauleit S, Sauerwein M (2016) Worum geht es bei Stadtökologie und ihrer Anwendungen in der Stadtentwicklung? In: Stadtökosysteme: Funktion, Management und Entwicklung. Springer, Heidelberg, Berlin Heidelberg, S245-254

19. Kaplan R, Kaplan S (1989) The experience of nature: a psychological perspective. Cambridge University Press, Cambridge

20. Frumkin $H$ (2001) Beyond toxicity: human health and the natural environment. Am J Prev Med 20:234-240

21. De Vries S, Verheij RA, Groenewegen PP, Spreeuwenberg P (2003) Natural environments - healthy environments? An exploratory analysis of the relationship between greenspace and health Environ Plan A 35:1717-1731

22. Maller C, Townsend M, Pryor A, Brown P, Leger StL (2006) Healthy nature healthy people: "contact with nature" as an upstream health promotion intervention for populations. Health Promot Int 21:45-54

23. Martens D, Bauer N (2011) Erholung in unterschiedlich genutzten Landwirtschaftsgebieten. Nat Landsch 86:307-311

24. De Keijzer C, Gascon M, Nieuwenhuijsen MJ, Dadvand $P$ (2016) Long-term green space exposure and cognition across the life course: a systematic review. Curr Environ Health Rep 3:468-477

25. Hartig T, Evans GW, Jamner LD, Davis DS, Gärling $T$ (2003) Tracking restoration in natural and urban field settings. JEnviron Psychol 23:109-123

26. Roe J, Aspinall P (2011) The restorative benefits of walking in urban and rural settings in adults with good and poor mental health. Health Place 17:103-113
27. Beyer KM, Kaltenbach A, Szabo A, Bogar S, Nieto FJ, Malecki KM (2014) Exposure to neighborhood green space and mental health: evidence from the survey of the health of Wisconsin. Int J Environ Res Public Health 11:3453-3472

28. Maas J, Verheij RA, De Vries S, Spreeuwenberg P, Schellevis FG, Groenewegen PP (2009) Morbidity is related to a green living environment. J Epidemiol Community Health 63:967-973

29. Alcock I, White MP, Wheeler BW, Fleming LE, Depledge MH (2014) Longitudinal effects on mental health of moving to greener and less green urban areas. Environ Sci Technol 48:1247-1255

30. Dallimer M, Irvine KN, Skinner AMJ et al (2012) Biodiversity and the feel-good factor: understanding associations between self-reported human well-being and species richness. Bioscience 62:47-55

31. Korpela KM, Ylen M, Tyrvainen L, Silvennoinen $H$ (2010) Favorite green, waterside and urban environments, restorative experiences and perceived health in Finland. Health Promot Int 25:200-209

32. Völker S, Kistemann T (2015) Developing the urban blue: comparative health responses to blue and green urban open spaces in Germany. Health Place 35:196-205

33. Tsunetsugu Y, Lee J, ParkB-J, Tyrväinen L, Kagawa T, Miyazaki Y (2013) Physiological and psychological effects of viewing urbanforestlandscapesassessed by multiple measurements. Landsc Urban Plan 113:90-93

34. Tyrväinen L, Ojala A, Korpela K, Lanki T, Tsunetsugu Y, Kagawa T (2014) The influence of urban green environments on stress relief measures: a field experiment. JEnviron Psychol 38:1-9

35. Kühn S, Duzel S, Eibich P et al (2017) In search of features that constitute an "enriched environment" in humans: Associations between geographical properties and brain structure. Sci Rep 7:11920

36. Ulrich RS (1984) View through a window may influence recovery from surgery. Science 224:420-421

37. Li Q, Morimoto K, Kobayashi M et al (2008) Visiting a forest, but not a city, increases human natural killer activity and expression of anticancer proteins. Int J Immunopathol Pharmacol 21:117-127

38. Bowler DE, Buyung-Ali LM, Knight TM, Pullin AS (2010) A systematic review of evidence for the added benefits to health of exposure to natural environments. BMC Public Health 10:456

39. Takano T, Nakamura K, Watanabe M (2002) Urban residential environments and senior citizens' longevity in megacity areas: the importance of walkable green spaces. J Epidemiol Community Health 56:913-918

40. Mitchell R, Popham F (2008) Effect of exposure to natural environment on health inequalities: an observational population study. Lancet 372:1655-1660

41. Cabe-Commission for Architecture and the Built Environment (Hrsg) (2010) Community green: using local spaces to tackle inequality and improve health. CABE, London

42. Richardson EA, Mitchell R (2010) Gender differences in relationships between urban green space and health in the United Kingdom. Soc Sci Med 71:568-575

43. Markevych I, Fuertes E, Tiesler CM et al (2014) Surrounding greenness and birth weight: results from the GINIplus and LISAplus birth cohorts in Munich. Health Place 26:39-46

44. Dadvand P, Sunyer J, Basagana X et al (2012) Surrounding greenness and pregnancy outcomes in four Spanish birth cohorts. Environ Health Perspect 120:1481-1487

45. Frank K, Frohn J, Härtich G et al (2004) Grün für Körperund Seele:ZurWertschätzung und Nutzung von Stadtgrün durch die Bielefelder Bevölkerung. Bielefeld 2000plus, Bielefeld

46. Maas J, Verheij RA, Spreeuwenberg P, Groenewegen PP (2008) Physical activity as a possible mechanism behind the relationship between green space and health: a multilevel analysis. BMC Public Health 8:206

47. Dadvand P, Bartoll X, Basagana Xet al (2016) Green spaces and general health: roles of mental health status, social support, and physical activity. Environ Int 91:161-167

48. De Vries S, Claßen T, Eigenheer-Hug SM et al (2011) Contributions of natural environments to physical activity. In: Nilsson K, Sangster M, Gallis C, Hartig T, de Vries S, Seeland K, Schipperijn J (Hrsg) Forests, trees and human health. Springer, Dordrecht, $\mathrm{S}$ 205-243

49. Maas J, Van Dillen SM, Verheij RA, Groenewegen PP (2009) Social contacts as a possible mechanism behind the relation between green space and health. Health Place 15:586-595

50. Strohmeier A, Mai U (2007) In guter Gesellschaft: Städtische Öffentlichkeit in Parks. Eine vergleichende Untersuchung von Nordpark und Bürgerpark in Bielefeld. Bielefeld 2000plus, Bielefeld

51. Ellaway A, Macintyre S, Bonnefoy X (2005) Graffiti, greenery, and obesity in adults: secondary analysis of European cross sectional survey. BMJ 331:611-612

52. Kuo FE, Sullivan WC (2001) Environment and crime in the inner city:does vegetation reduce crime? Environ Behav 33:343-367

53. Müller C, Brückner H, Dietrich C et al (2016) Stadtnatur fördert sozialen Zusammenhalt. In: Kowarik I, Bartz R, Brenck M (Hrsg) Ökosystemleistungen in derStadt-Gesundheit schützen und Lebensqualität erhöhen. Naturkapital Deutschland - TEEB DE, Berlin, Leipzig, S126-145

54. Claßen T, Heiler A, Brei B (2012) Urbane Grünräume und gesundheitliche Chancengleichheit längst nicht alles im „grünen Bereich“. In: Bolte $G$, Bunge $\mathrm{C}$, Hornberg C, Köckler H, Mielck A (Hrsg) Umweltgerechtigkeit durch Chancengleichheit bei Umwelt und Gesundheit: Konzepte, Datenlage und Handlungsperspektiven. Huber, Bern, S113-123

55. Van Dillen SM, De Vries $S$, Groenewegen PP, Spreeuwenberg P (2012) Greenspace in urban neighbourhoods and residents' health: adding quality to quantity. J Epidemiol Community Health 66:e8

56. De Vries S, Van Dillen SM, Groenewegen PP, Spreeuwenberg P (2013) Streetscape greenery and health: stress, social cohesion and physical activity as mediators. Soc Sci Med 94:26-33

57. Francis J, Wood LJ, Knuiman M, Giles-Corti B (2012) Quality or quantity? Exploring the relationship between Public Open Space attributes and mental health in Perth, Western Australia. Soc Sci Med 74:1570-1577

58. Churkina G, Kuik F, Bonn B et al (2017) Effect of VOC emissions from vegetation on air quality in Berlin during a heatwave. Environ Sci Technol 51:6120-6130

59. Bixler RD, Floyd MF (1997) Nature is scary, disgusting, and uncomfortable. Environ Behav 29:443-467 


\section{Leitthema}

60. Umweltbundesamt (Hrsg) (2016) Hintergrundpapier Eichenprozessionsspinner. Antworten auf häufig gestellte Fragen. UBA, Dessau/Berlin

61. Schmitz R, KuhnertR, Thamm M (2017) 12-MonatsPrävalenz von Allergien in Deutschland. J Health Monit 2(1):77-82. https://doi.org/10.17886/RKLGBE-2017-011.2

62. Dadvand $P$, Villanueva $C M$, Font-Ribera $L$ et al (2014) Risks and benefits of green spaces for children: a cross-sectional study of associations with sedentary behavior, obesity, asthma, and allergy. Environ Health Perspect 122:1329

63. Bergmann K-C, Zuberbier T, Augustin J, Mücke H-G, Straff W (2012) Klimawandel und Pollenallergie: Städte und Kommunen sollten bei der Bepflanzung des öffentlichen Raums Rücksicht auf Pollenallergiker nehmen. Allergo J 21:103-108

64. BeckerC,HübnerS, KrügerT, KreutzS (2017) Urbane Freiräume: Qualifizierung, Rückgewinnung und Sicherung urbaner Frei- und Grünräume Endbericht (September 2017). Bundesinstitut für Bau-, Stadt-und Raumforschung (BBSR, Berlin

65. BMUB - Bundesministerium für Umwelt, $\mathrm{Na}$ turschutz, Bau und Reaktorsicherheit (2017) Weißbuch Stadtgrün: Grün in der Stadt - Für eine lebenswerte Zukunft. BMUB, Berlin 